\title{
The maximum angle condition is not necessary for convergence of the finite element method
}

\author{
Antti Hannukainen ${ }^{1}$, Sergey Korotov ${ }^{2}$, Michal Křřžek ${ }^{3}$
}

October 19, 2010

\author{
${ }^{1}$ Department of Mathematics and Systems Analysis, Aalto University \\ P.O. Box 11100, FI-00076 Aalto, Finland \\ e-mail: antti.hannukainen@aalto.fi \\ 2 BCAM - Basque Center for Applied Mathematics \\ Bizkaia Technology Park, Building 500, E-48160 Derio \\ Basque Country, Spain \\ e-mail: korotov@bcamath.org \\ ${ }^{3}$ Institute of Mathematics, Academy of Sciences \\ Žitná 25, CZ-115 67 Prague 1, Czech Republic \\ e-mail: krizek@math.cas.cz
}

\begin{abstract}
We show that the famous maximum angle condition in the finite element analysis is not necessary to achieve the optimal convergence rate when simplicial finite elements are used to solve elliptic problems. This condition is only sufficient. In fact, finite element approximations may converge even though some dihedral angles of simplicial elements tend to $\pi$.
\end{abstract}

Keywords: finite element method, Céa's lemma, maximum angle condition, Lagrange and Hermite simplicial finite elements, red simplicial refinement, nested triangulations.

Mathematical Subject Classification: 65N30, 65N50, 65N12

\section{Introduction}

Angle conditions have several important roles in the analysis of the finite element method. They enable us to derive the optimal order interpolation bounds and prove convergence of the finite element method, to derive various a posteriori error estimates, to perform regular mesh refinements, to preserve qualitative properties of smooth solutions in FE simulations, etc. Note that the only one obtuse triangle in a triangulation can completely destroy the discrete maximum principle (see [7, p. 329]).

In order to clarify the situation with the convergence of the finite element method in the context of angle conditions, we consider a family $\mathcal{F}=\left\{\mathcal{T}_{h}\right\}_{h \rightarrow 0}$ of face-to-face triangulations of a polygonal domain into closed triangles. In 1968 Miloš Zlámal [19] 
introduced the following minimum angle condition which states that there should exist a constant $\alpha_{0}$ such that for any triangulation $\mathcal{T}_{h} \in \mathcal{F}$ and any triangle $K \in \mathcal{T}_{h}$ we have

$$
0<\alpha_{0} \leq \alpha_{K}
$$

where $\alpha_{K}$ is the minimal angle of $K$. Under this (sufficient) condition he derived the optimal order bounds of the interpolation error in the Sobolev $H^{1}$-norm (and $H^{2}$-norm) and therefore also of the discretization error for the finite element method applied to second (and fourth) order elliptic equation with some boundary conditions. The same condition was also introduced by Alexander Ženíšek [17] for the finite element method applied to a system of linear elasticity equations of second order, published in 1969. However, this paper was submitted already on April 3, 1968, whereas Zlámal's paper on April 17, 1968. Nevertheless, condition (1) is known as Zlámal's minimum angle condition, since [17] was published in Czech. For a generalization of the minimum angle condition into three-dimensional case see [4], and for higher dimensions see e.g. [5, 6, 8].

In 1976, three research groups (see $[2,3,11]$ ) independently found that condition (1) can be weakened to prove the optimal rate of the interpolation error which by the wellknown Céa's lemma yields also the optimal rate of the discretization error of the finite element method. They derived the so-called maximum angle condition: There exists a constant $\gamma_{0}$ such that for any triangulation $\mathcal{T}_{h} \in \mathcal{F}$ and any triangle $K \in \mathcal{T}_{h}$ we have

$$
\gamma_{K} \leq \gamma_{0}<\pi
$$

where $\gamma_{K}$ is the maximum angle of $K$.

Clearly, (1) implies (2), since $\gamma_{K} \leq \pi-2 \alpha_{K} \leq \pi-2 \alpha_{0} \equiv \gamma_{0}$, but the converse implication does not hold.

Note that John L. Synge [16] already in 1957 proved the optimal order of nodal linear interpolation under condition (2). This condition was later generalized in various directions, e.g., to three dimensions (see [13]), to general Sobolev norms $\|\cdot\|_{k, p}($ for $p \neq 2)$ [12], to anisotropic meshes [1], etc.

In $[2$, p. 223], $[15$, p. 138], and $[17$, p. 365] there are examples showing that if the maximum angle condition (2) does not hold then the linear triangular finite elements loose their optimal interpolation order. The main idea of all these examples is the following.

Take $\varepsilon>0$ and the triangle $K$ with vertices $A_{1}=(-1,0), A_{2}=(1,0)$, and $A_{3}=(0, \varepsilon)$ (see Figure 1). Consider the function $v\left(x_{1}, x_{2}\right)=x_{1}^{2}$ and its linear interpolant

$$
\left(L_{\varepsilon} v\right)\left(x_{1}, x_{2}\right)=-\frac{x_{2}}{\varepsilon}+1 \quad \text { on } K,
$$

i.e.,

$$
\left(L_{\varepsilon} v\right)\left(A_{i}\right)=v\left(A_{i}\right), \quad i=1,2,3 .
$$

Using the standard Sobolev space notation, (3), and the fact $\frac{\partial v}{\partial x_{2}}=0$, we find that

$$
\left\|v-L_{\varepsilon} v\right\|_{1, K}^{2} \geq\left|\frac{\partial L_{\varepsilon} v}{\partial x_{2}}\right|_{0, K}^{2}=\frac{1}{\varepsilon^{2}} \text { meas } K=\frac{1}{\varepsilon} \rightarrow \infty \quad \text { as } \varepsilon \rightarrow 0 \quad \text { and } \gamma_{K} \rightarrow \pi .
$$

We conclude that one badly shaped triangle in every triamgulation $\mathcal{T}_{h} \in \mathcal{F}$ can yield an arbitrary large interpolation error in the Sobolev $H^{1}$-norm. 


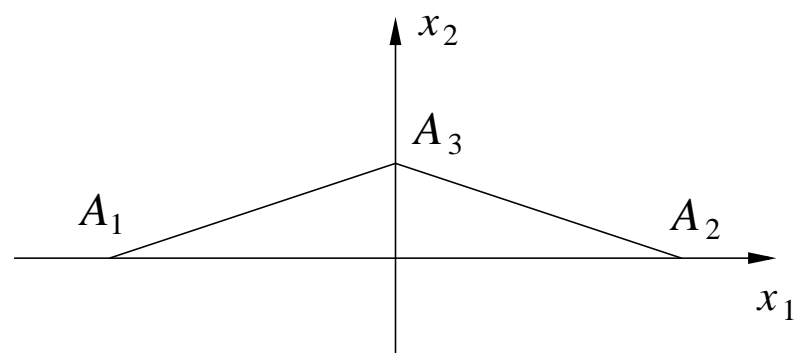

Figure 1: Degenerating triangle for $\gamma_{K} \rightarrow \pi$.

For tetrahedral elements similar examples can also be constructed, see [13, p. 518]. Namely, if the maximal angle between two faces or the maximal angle between edges tends to $\pi$, then the interpolation error may tend to $\infty$ like in (4).

Examples similar to (3)-(4) caused numerical analysts to believe that large dihedral angles of simplicial elements (i.e., when the maximum angle condition (2) is not satisfied) produce also large discretization error when solving second order elliptic problems by the finite element method. For instance, Babuška and Aziz [2] state that the maximum angle condition (2) is essential for convergence of the finite element method, whereas D'Azevedo and Simpson [9, p. 1063] assert that (2) is necessary and sufficient for convergence. To the contrary, in this paper we show that the finite element method may converge even when $(2)$ is violated.

Let us emphasize that the Céa's lemma gives only an upper bound of the discretization error by means of the interpolation error. Note that the discretization error is, in some cases, of the same order as the interpolation error. This was proved e.g. for uniform triangulations that satisfy the minimum angle condition (1) for a second order elliptic equation with smooth variable coefficients (see [14]). But in general, the discretization error can be much smaller than the interpolation error, as we will later demonstrate (see the right of Figure 4).

In Section 2 we give illustrative two-dimensional examples showing that the practical convergence rate of the discretization error seems to be of optimal order (i.e. very small) even though the maximal angle over all triangles tends to $\pi$ like in (4), i.e., the maximum angle condition is not necessary. In Section 3 we generalize this example to simplicial elements of an arbitrary space dimension. Finally, in Section 4 we present some numerical results for the red refinement algorithm for tetrahedral partitions.

\section{Why is the maximum angle condition not neces- sary?}

Keeping in mind the result (4), we now show that the discretization error can be very small, whereas the interpolation error is large. For simplicity, consider the Poisson equation with the homogeneous Dirichlet boundary conditions in the unit square $\Omega=(0,1) \times(0,1)$,

$$
-\Delta u=f \quad \text { in } \quad \Omega, \quad u=0 \quad \text { on } \partial \Omega,
$$

where $f \in L^{2}(\Omega)$. Since $\Omega$ is convex, its weak solution is from the Sobolev space $H^{2}(\Omega)$ $[10]$ and thus continuous by the Sobolev imbedding theorem. 
Example 1: We will define two special families $\mathcal{F}_{1}$ and $\mathcal{F}_{2}$ of nested triangulations of $\bar{\Omega}$. To this end we first introduce uniform rectangular meshes of the given unit square consisting of congruent rectangles. Its horizontal sides will be divided into $2^{k}$ equal parts and the vertical parts will be divided into $4^{k}$ equal parts for $k=0,1,2, \ldots$ To construct the family $\mathcal{F}_{1}$ we divide each rectangle by its diagonal with a positive slope (see Figure 2), whereas for the family $\mathcal{F}_{2}$ we take both diagonals (see Figure 3 ). We observe that the first family $\mathcal{F}_{1}$ satisfies the maximum angle condition $(2)$ with $\gamma_{0}=\pi / 2$ for all $k$, whereas for the second family $\mathcal{F}_{2}$ we observe that $\gamma_{K} \rightarrow \pi$ for every second triangle. Let $V_{h}$ and $W_{h}$ be finite element spaces of continuous and piecewise linear functions over triangulations from $\mathcal{F}_{1}$ and $\mathcal{F}_{2}$, respectively. Obviously,

$$
V_{h} \subset W_{h} .
$$

Denote by $u_{h} \in W_{h}$ the standard Galerkin approximation of the weak solution $u$ of (5). Let $L_{h} u$ stands for the linear interpolant of $u$ in $V_{h}$. Then by Céa's lemma (see [8]) there exists a constant $C>0$ such that

$$
\left\|u-u_{h}\right\|_{1} \leq C \inf _{w_{h} \in W_{h}}\left\|u-w_{h}\right\|_{1} \leq C \inf _{v_{h} \in V_{h}}\left\|u-v_{h}\right\|_{1} \leq C\left\|u-L_{h} u\right\|_{1} \leq C^{\prime} h|u|_{2} \text { as } h \rightarrow 0
$$

where the last inequality can be proved under the assumption (2) (see e.g. [2, 11, 12]) for another constant $C^{\prime}>0$ independent of the discretization parameter $h$. This example shows that the discretization error tends to 0 at least linearly in the $H^{1}$-norm even though the maximal angle of every second triangle from any $\mathcal{T}_{h} \in \mathcal{F}_{2}$ tends to $\pi$. In Figure 4 we observe the practical rates of convergence on $\mathcal{F}_{1}$ and $\mathcal{F}_{2}$ for problem (5) with the following right-hand side

$$
f\left(x_{1}, x_{2}\right)=\pi^{2} \sin \pi x_{1} \sin \pi x_{2} .
$$
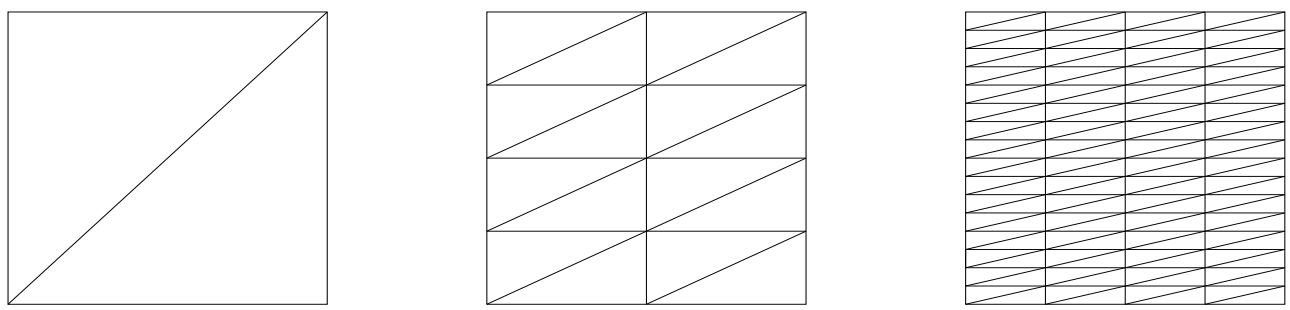

Figure 2: Family $\mathcal{F}_{1}$ satisfying the maximum angle condition.
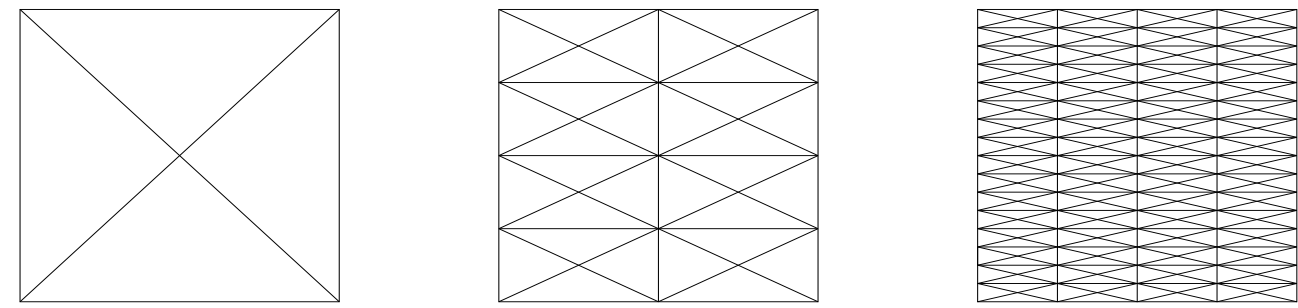

Figure 3: Family $\mathcal{F}_{2}$ that does not satisfy the maximum angle condition. 

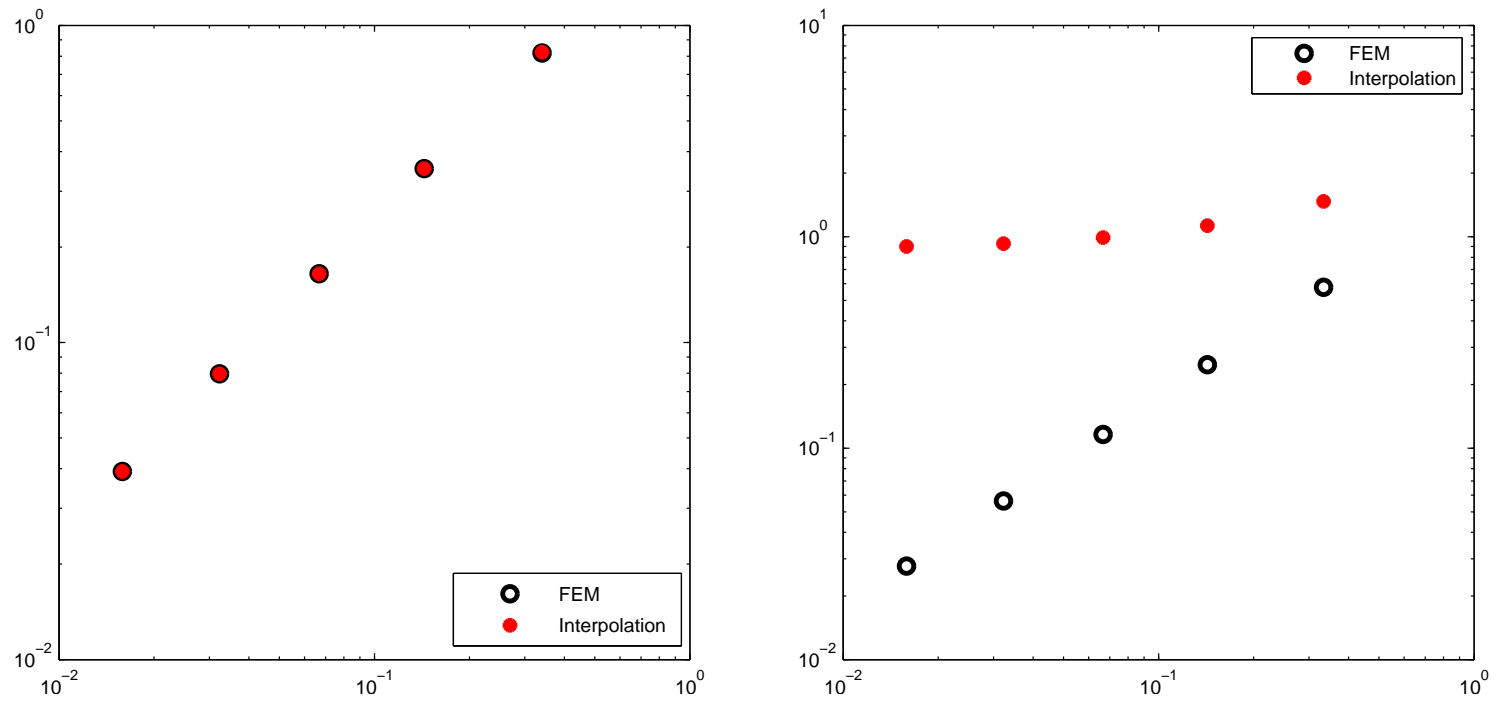

Figure 4: The practical convergence rates for the families $\mathcal{F}_{1}$ (left) and $\mathcal{F}_{2}$ (right). The horizontal axis corresponds to the discretization parameter and the vertical axis corresponds to the $H^{1}$-norm of the discretization and interpolation errors. The difference between interpolation and discretization errors on the left figure is very small, which cannot be seen from the graph.

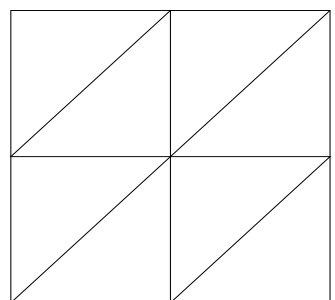

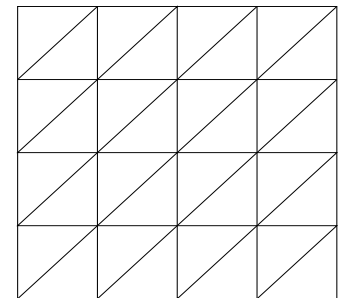

Figure 5: Family $\mathcal{F}_{3}$ satisfying the minimum angle condition. 

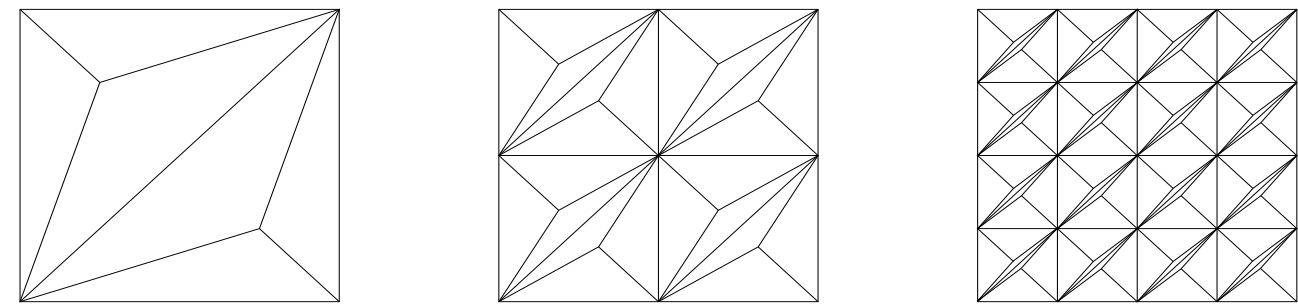

Figure 6: Family $\mathcal{F}_{4}$ that does not satisfy the maximum angle condition.

\section{Some generalization of two-dimensional examples to arbitrary space dimension}

Let the unit $d$-cube $\Omega=(0,1)^{d}, d \in\{2,3, \ldots\}$, be divided uniformly into congruent $d$-blocks. Consider for instance the $d$-block

$$
B=\left(0, h_{1}\right) \times \cdots \times\left(0, h_{d}\right) .
$$

Without loss of generality we may assume that

$$
h_{1} \geq h_{2} \geq \cdots \geq h_{d}
$$

where $h_{i}^{-1}$ is integer for $i \in\{1, \ldots, d\}$. Moreover, let

$$
h_{1}=h_{1}(k)=2^{-k} \text { and } h_{d}=h_{d}(k)=4^{-k} \text { for } k=0,1,2, \ldots
$$

We will again consider two families $\mathcal{F}_{5}$ and $\mathcal{F}_{6}$ of nested simplicial partitions. Partitions from $\mathcal{F}_{5}$ are based on Kuhn's partition (see [7]). For instance, if $k=0$ then $\bar{\Omega}$ is decomposed into $d$ ! nonobtuse simplices defined as follows

$$
K_{\sigma}=\left\{x=\left(x_{1}, \ldots, x_{d}\right) \in \mathbf{R}^{d} \mid 0 \leq x_{\sigma(1)} \leq \cdots \leq x_{\sigma(d)} \leq 1\right\},
$$

where $\sigma$ ranges over all permutations of the numbers $1,2, \ldots, d$. For $k \geq 1$ all the resulting $d$-blocks are decomposed into $d$-simplices in a topologically similar way. None of the dihedral angles of these simplices is greater than $\pi / 2$.

To define the family $\mathcal{F}_{6}$ we denote by $G$ the centre of gravity of each $d$-block. Consider again Kuhn's partition of each $(d-1)$-dimensional facet of a given $d$-block. Now we take the convex hull of $G$ and each $(d-1)$-dimensional simplex from the block boundary. This gives required $d$-simplices. Some of them contain large dihedral angles tending to $\pi$ as $k \rightarrow \infty$.

To show this, we can consider, without loss of generality, the nonobtuse $d$-simplex with vertices $A_{0}=(0,0, \ldots, 0), A_{1}=(1,0, \ldots, 0), \ldots, A_{d-1}=(1, \ldots, 1,0)$, and $A_{d}=$ $(1, \ldots, 1, \varepsilon)$, with $\varepsilon$ tending to zero.

Introducing the mid-point $G=\left(\frac{1}{2}, \ldots, \frac{1}{2}, \frac{\varepsilon}{2}\right)$ of the longest edge, we see that the subsimplex $A_{0} A_{1} \ldots A_{d-1} G$ is from the family $\mathcal{F}_{2}$ (up to scaling). Now, the hyperplane containing its facet $A_{0} A_{1} \ldots A_{d-2} G$ is described by the following equation:

$$
-\varepsilon x_{d-1}+x_{d}=0
$$

and the one containing the adjacent facet $A_{1} A_{2} \ldots A_{d-1} G$ is of the form

$$
\varepsilon x_{1}+x_{d}-\varepsilon=0 .
$$


The angle $\gamma_{\varepsilon}$ between these two hyperfaces can be calculated via scalar product of their normals. This gives $\cos \left(\pi-\gamma_{\varepsilon}\right)=1 /\left(1+\varepsilon^{2}\right)$ that tends to 1 as $\varepsilon \rightarrow 0$, which means that the angle $\gamma_{\varepsilon}$ between the chosen facets tends to $\pi$.

We can now consider problem (5) in arbitrary space dimension. If its solution is smooth enough, the Lagrange interpolation operator is well defined and (6) holds again.

In fact, a more universal statement, applicable also for nonsimplicial elements, can be formulated as follows. Consider a general elliptic problem in a weak form: Find $u \in V$ such that

$$
a(u, v)=F(v) \quad \forall v \in V,
$$

where $V$ is a Hilbert space, $a(\cdot, \cdot)$ is a continuous $V$-elliptic bilinear form, and $F(\cdot)$ is a linear continuous functional over $V$, see [8]. Then we have:

Theorem 1 Let $\left\{V_{h}\right\}_{h \rightarrow 0}$ and $\left\{W_{h}\right\}_{h \rightarrow 0}$ be two families of spaces of piecewise polynomial finite element functions such that $V_{h} \subset W_{h} \subset V$. Assume that for each $v \in V$

$$
\lim _{h \rightarrow 0} \inf _{v_{h} \in V_{h}}\left\|v-v_{h}\right\|_{V}=0,
$$

i.e., the union $\bigcup_{h>0} V_{h}$ is dense in $V$. Then

$$
\left\|u-u_{h}\right\|_{V} \rightarrow 0
$$

where $u_{h} \in W_{h}$ is the standard finite element approximation of the weak solution $u \in V$ of elliptic boundary value problem (8).

P r o o f : From Cea's lemma and (9), we obtain

$$
\left\|u-u_{h}\right\|_{V} \leq C \inf _{w_{h} \in W_{h}}\left\|u-w_{h}\right\|_{V} \leq C \inf _{v_{h} \in V_{h}}\left\|u-v_{h}\right\|_{V} \rightarrow 0 \text { as } h \rightarrow 0 .
$$

\section{Red refinement techniques}

Let $\Omega=(0,1)^{3}$ and consider the problem

$$
-\Delta u=\sin \pi x \sin \pi y \sin \pi z \quad \text { in } \quad \Omega \text { and } \quad u=0 \text { on } \partial \Omega .
$$

The initial mesh is Kuhn's division of the cube into six nonobtuse tetrahedra (see (7)). In [18] Zhang proposes a special kind of red refinement of tetrahedral partitions that does not produce large dihedral angles tending to $\pi$.

Consider the standard red refinement algorithm for a tetrahedron into eight subtetrahedra, using two different strategies, the longest-diagonal and shortest-diagonal refinement, for dividing the interior octahedron (see Figure 7).

In the longest-diagonal refinement, the interor octahedron is divided by taking the longest diagonal as a common edge for all resulting subtetrahedra. In the shortest-diagonal refinement, the shortest edge is chosen as the common edge. This will lead to considerably slower decay of $h$, the longest edge in the mesh, in comparison to the shortest-diagonal refinement. In practice, this means that certain value of $h$ is obtained for smaller number of 


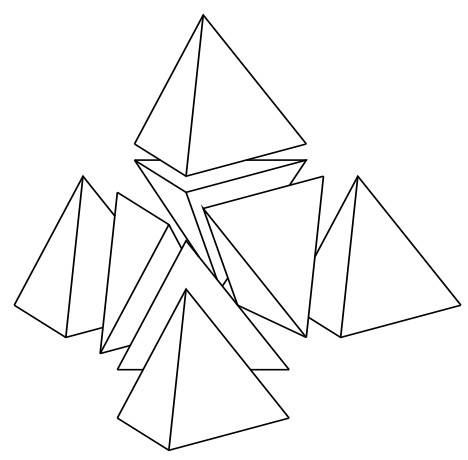

Figure 7: Red refinement of a tetrahedron.

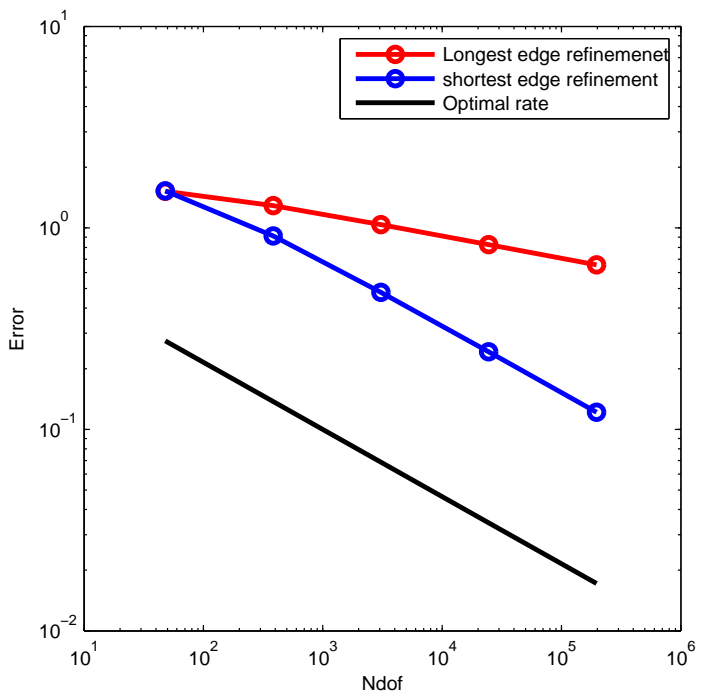
refinement. different. of degrees of freedom in the mesh. degrees of freedom for the shortest-diagonal refinement, compared to the longest-diagonal

In some sense using $h$ as a measure for convergence is not correct in this example. Although the optimal $\mathcal{O}(h)$ convergence rate would be obtained, the number of degrees of freedom required to achieve the same accuracy for the both algorithms would be totally

In Figure 8, we have visualized the convergence rate in the $H^{1}$-norm for the problem (10). The initial partition is again Kuhn's division of the cube into six nonobtuse tetrahedra (see $(7)$ ). The practical rate of convergence for the longest-diagonal refinement is about $h^{1 / 2}$ and for the shortest-diagonal refinement $h$. However, when degrees of freedom are compared, the longest-diagonal refinement performs considerably worse. In this case, the shortest-diagonal refinement seems to have the practical convergence rate of $\mathcal{O}\left(N^{-1 / 3}\right)$, whereas the longest-diagonal refinement $\mathcal{O}\left(N^{-1 / 10}\right)$, where $N$ is the number

Figure 8: The $H^{1}$-norm of the discretization error versus the number of degrees of freedom and the discretization parameter.

This example shows us that sometimes large angles really do matter, i.e., the maximum

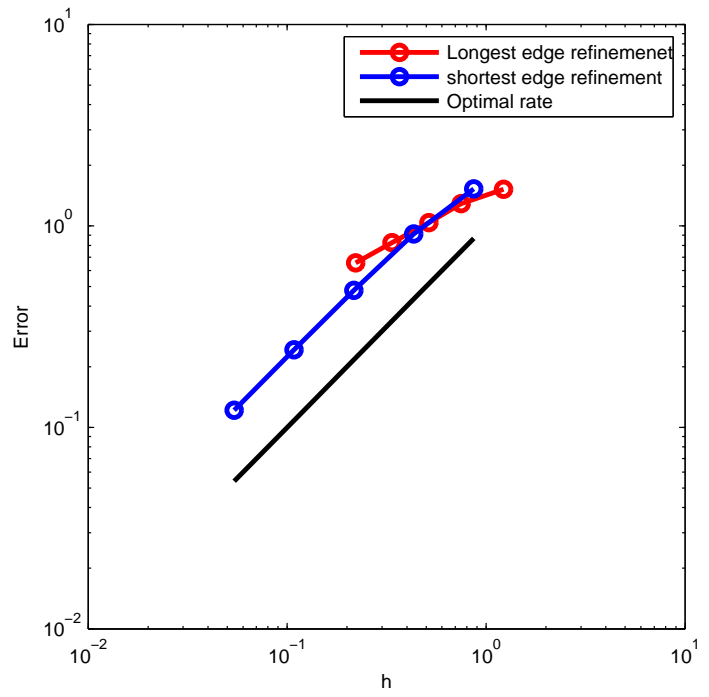
angle condition is essential, even though it is not necessary. 
Acknowledgement: This work was supported by Grant MTM2008-03541 of the MICINN, Spain, the ERC Advanced Grant FP7-246775 NUMERIWAVES, Grant PI2010-04 of the Basque Government, and Grant no. IAA 100190803 of the Academy of Sciences of the Czech Republic. The authors are also thankful to Jan Brandts for fruitful discussions.

\section{References}

[1] T. Apel, M. Dobrowolski. Anisotropic interpolation with applications to the finite element method. Computing 47 (1992), 277-293.

[2] I. Babuška, A. K. Aziz. On the angle condition in the finite element method. SIAM J. Numer. Anal. 13 (1976), 214-226.

[3] R. E. Barnhill, J. A. Gregory. Sard kernel theorems on triangular domains with applications to finite element error bounds. Numer. Math. 25 (1976), 215-229.

[4] J. Brandts, S. Korotov, M. Křížek. On the equivalence of regularity criteria for triangular and tetrahedral finite element partitions. Comput. Math. Appl. 55 (2008), $2227-2233$.

[5] J. Brandts, S. Korotov, M. Křižzek. On the equivalence of ball conditions for simplicial finite elements in $R^{d}$. Appl. Math. Lett. 22 (2009), 1210-1212.

[6] J. Brandts, S. Korotov, M. Kř́žžek. Generalization of the Zlámal condition for simplicial finite elements in $R^{d}$. Appl. Math. 56 (2011), to appear.

[7] J. Brandts, S. Korotov, M. Křižzek, J. Šolc. On nonobtuse simplicial partitions. SIAM Rev. 51 (2009), 317-335.

[8] P. G. Ciarlet. The Finite Element Method for Elliptic Problems. North-Holland, Amsterdam, 1978.

[9] E. F. D'Azevedo, R. B. Simpson. On optimal interpolation triangle incidences. SIAM J. Sci. Statist. Comput. 10 (1989), 1063-1075.

[10] P. Grisvard. Elliptic Problems in Nonsmooth Domains. Pitman Monographs and Surveys in Pure and Applied Mathematics, vol. 24, Harlow, 1985.

[11] P. Jamet. Estimation de l'erreur pour des éléments finis droits presque dégénérés. RAIRO Anal. Numér. 10 (1976), 43-60.

[12] M. Křížek. On semiregular families of triangulations and linear interpolation. Appl. Math. 36 (1991), 223-232.

[13] M. Kř́žžek. On the maximum angle condition for linear tetrahedral elements. SIAM J. Numer. Anal. 29 (1992), 513-520.

[14] M. Křižzek, H.-G. Roos, W. Chen. Two-sided bounds of the discretization error for finite elements, (submitted in 2009), 10 pp. 
[15] G. Strang, G. Fix. An Analysis of the Finite Element Method. Prentice-Hall, Inc., Englewood Cliffs, New Jersey, 1973.

[16] J. L. Synge. The Hypercircle in Mathematical Physics. Cambridge Univ. Press, Cambridge, 1957.

[17] A. Ženíšek. The convergence of the finite element method for boundary value problems of a system of elliptic equations (in Czech). Apl. Mat. 14 (1969), 355-377.

[18] S. Zhang. Successive subdivisions of tetrahedra and multigrid methods on tetrahedral meshes. Houston J. Math. 21 (1995), 541-556.

[19] M. Zlámal. On the finite element method. Numer. Math. 12 (1968), 394-409. 